Phase Transforming Cellular Material (PXCM)

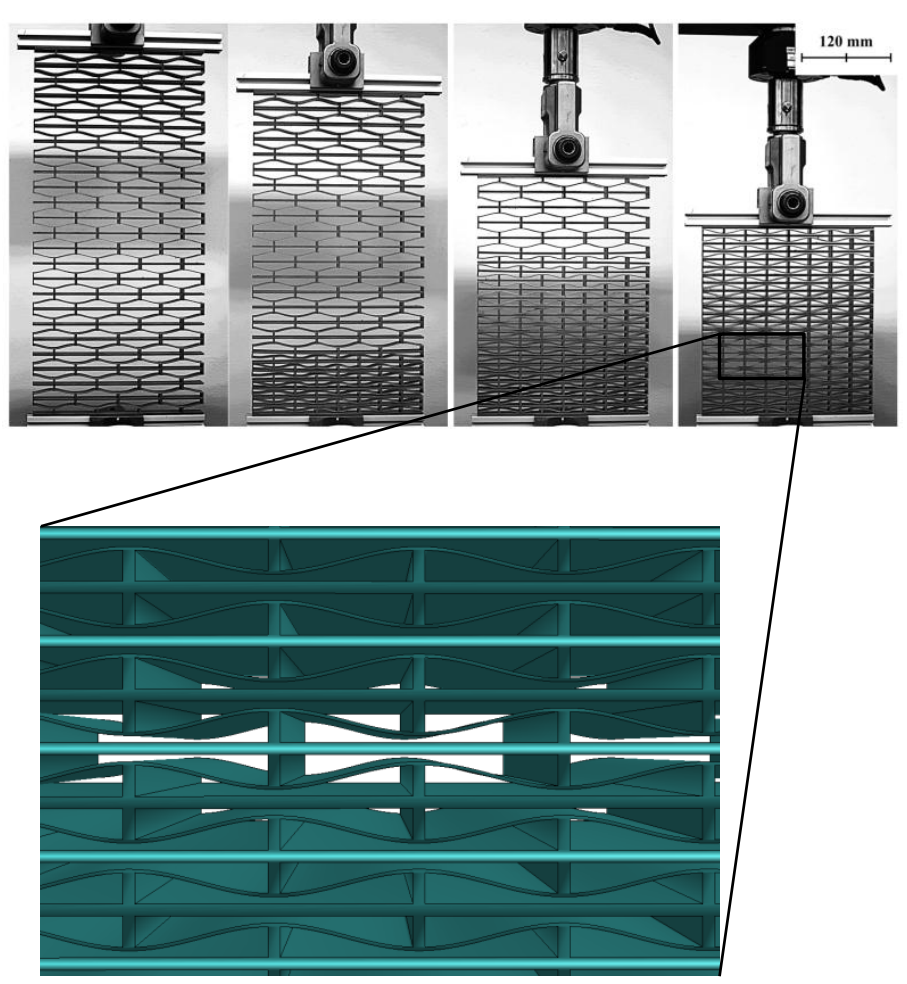

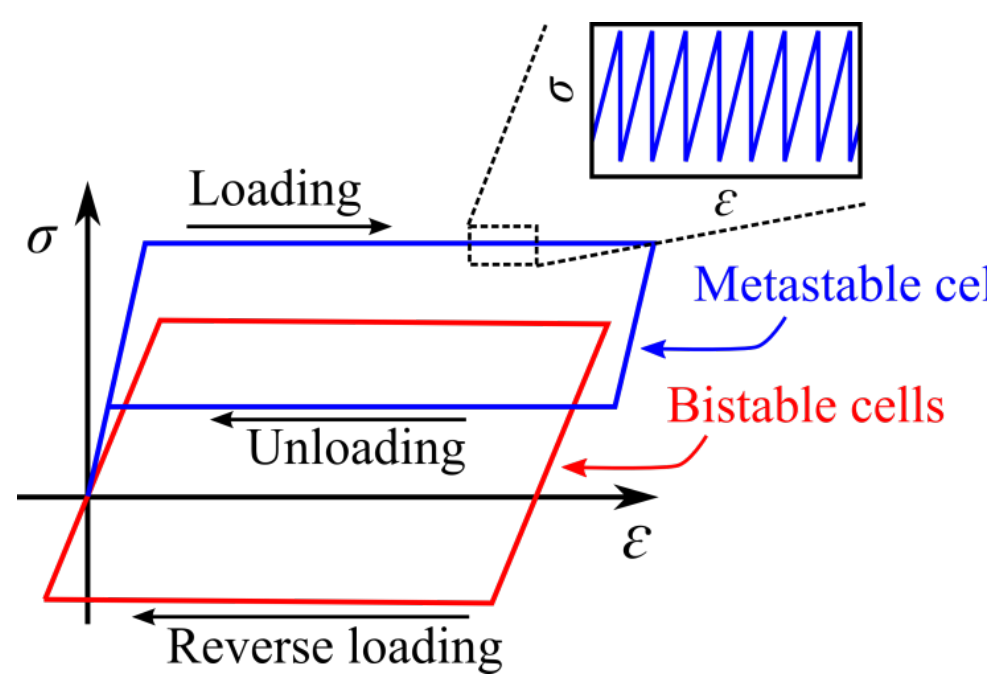

Mechanical Response and hysteretic behavior

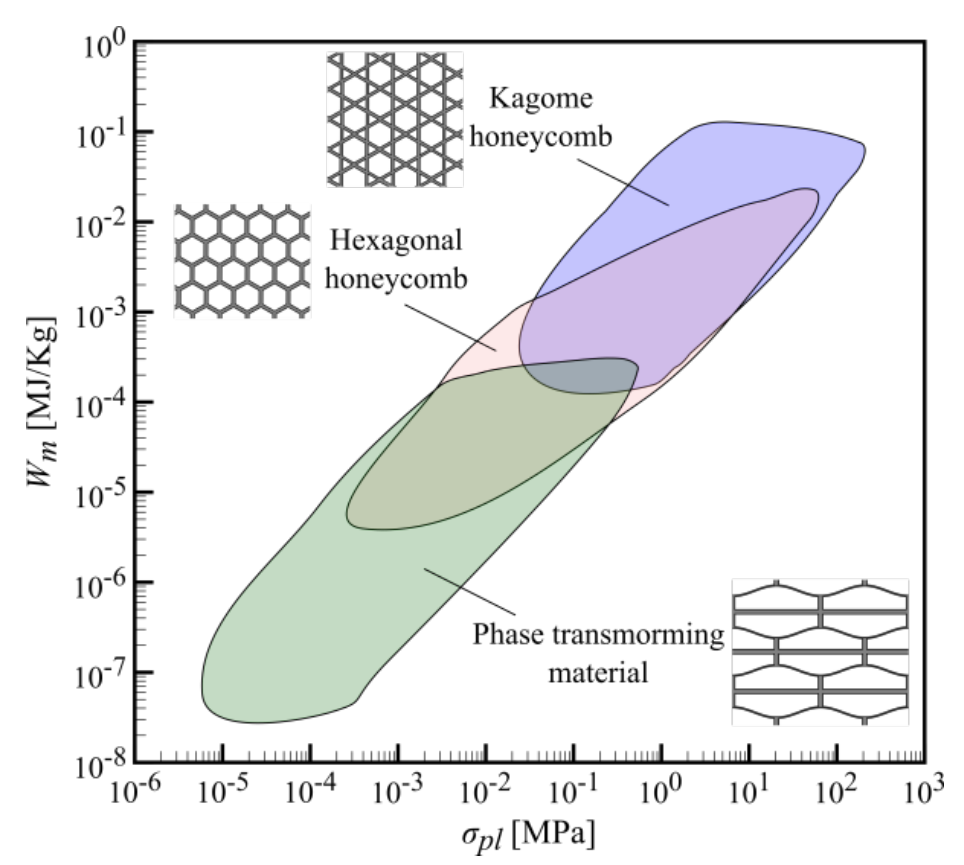

Comparison with other cellular materials 


\title{
Phase Transforming Cellular Materials
}

\author{
David Restrepo $^{1}$, Nilesh D. Mankame ${ }^{2}$, Pablo D. Zavattieri ${ }^{1^{*}}$ \\ ${ }^{1}$ Lyles School of Civil Engineering, Purdue University, West Lafayette, IN 47907, USA \\ ${ }^{2}$ Smart Materials and Structures, General Motors Global Research \& Development, Warren, MI 48090
}

\begin{abstract}
We extend the notion of phase transformations to periodic cellular materials by introducing materials whose unit cells have multiple stable configurations. Each stable configuration of the unit cell corresponds to a stable phase, and transitions between these phases are regarded as phase transformations of the cellular material. We present a cellular material that exhibits phase transformation, identify key elements of the unit cell geometry that yield its mechanical behavior and introduce an analytical model that describes its constitutive behavior. Cellular materials that exhibit phase transformations show hysteresis and their response is characterized by a long serrated loading and unloading plateaus, making these materials attractive for energy absorption applications. While these phase transforming cellular material offer an energy absorption performance that is comparable to metal and polymeric honeycombs, the key difference is that the deformation on these materials is fully recoverable.
\end{abstract}

Keywords: Cellular materials, phase transformation, energy absorption, compliant bistable/metastable mechanism.

\section{INTRODUCTION}

Active materials like shape memory, ferroelectric and magnetostrictive alloys obtain their characteristic properties due to phase transformations[1]-[4]. In these materials, phase transformations occur by changing the packing arrangement of the atoms in a process that resembles multistable mechanisms switching between stable configurations [5], [6]. A similar behavior has been observed in folded proteins in which phase transformations (i.e. the change from folded to unfolded configuration) provide the mechanisms through which biological materials obtain remarkable properties like combinations of strength and toughness, superelasticity, shock energy dissipation, among others[7]-[10].

In this work, we extend the notion of phase transforming materials to cellular materials whose base material does not necessarily exhibit phase transformations at the atomic level. To this end, we define a phase transformation in a cellular material as the change in geometry of its unit cell from one stable configuration to another stable (or metastable) configuration while keeping its original topology. The capability of a cellular material to undergo phase transformation is attained mainly by a proper choice of an elastic base material and, the topological and geometrical design of the unit cell in order to allow the elastic reversibility of the transformation. We present a phase transforming cellular material (PXCM)

\footnotetext{
${ }^{*}$ Corresponding author. Tel: +1 (765) 496-9644, Fax: +1 (765) 494-0395,

E-mail address: zavattie@purdue.edu (P.D. Zavattieri)
} 
whose unit cells comprise compliant bistable or metastable mechanisms (see the schematic representations in Fig. 1(a)-(b)). Such mechanisms exhibit a force-displacement behavior characterized by two limit points $\left(\left(d_{I}, F_{I}\right)\right.$ and $\left.\left(d_{I I}, F_{I I}\right)\right)$ that lead to three regimes in their mechanical response: Regimes I and III are characterized by a positive stiffness as they represent the deformation of stable configurations of the unit cell. These configurations correspond to local minima in the potential energy response of the mechanism. Regime II is characterized by a negative stiffness and corresponds to a transition of the mechanism from one limit point to another [11], [12]. In the case of a metastable mechanism, the second stable configuration corresponds to a special case of stability in which a sufficiently large disturbance along a specific direction can cause the mechanism to revert to its first stable configuration, which has a lower potential energy (Fig. 1(b))[13]. A metastable mechanism in the unit cell of a PXCM allows the material to undergo large deformations and return to its original configuration once the load is released. This behavior is similar to the pseudo-elasticity exhibited by superelastic shape memory alloys. On the other hand, a material with a bistable mechanism unit cell exhibits two stable phases. A region of the PXCM is said to be in phase 1 when all its cells are in the first stable/metastable configuration; and similarly for phase 2 .

(a)

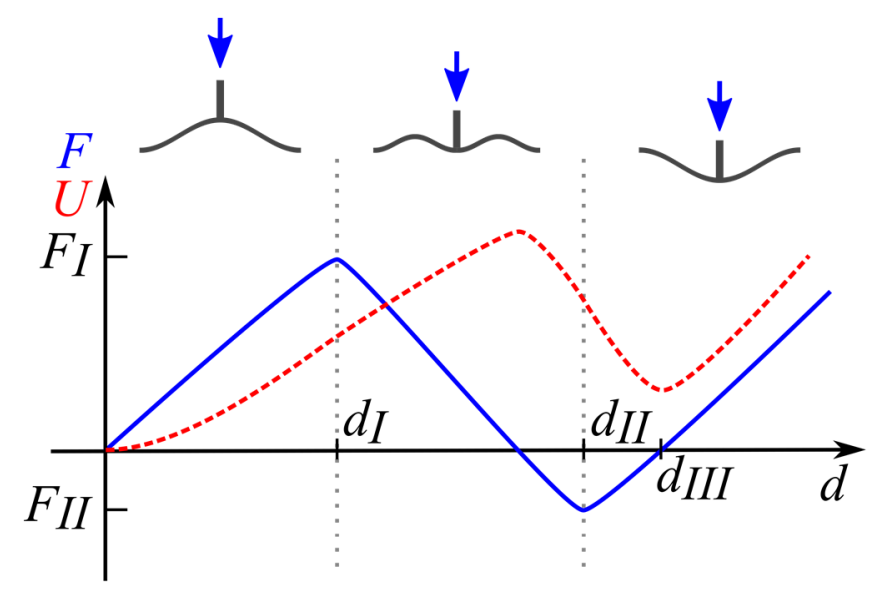

(b)

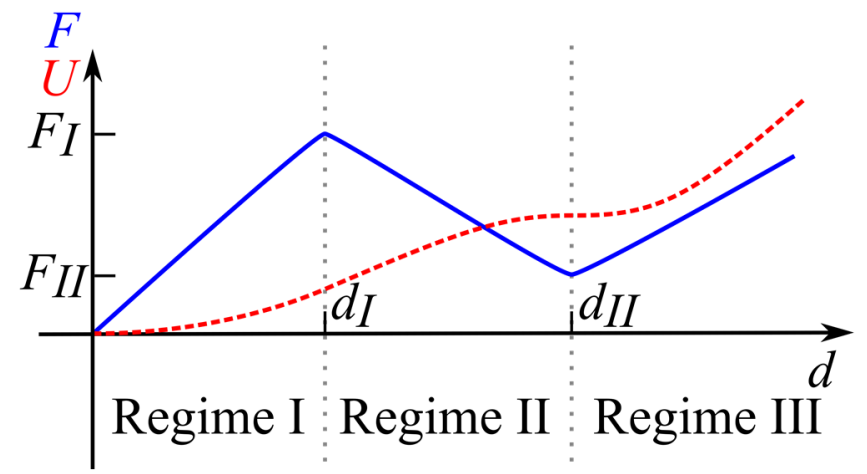

Fig. 1. Bistable or metastable behavior can be achieved by changing the geometrical parameters of the base mechanism. (a) Schematic representations for the force-displacement $(F-d)$ behavior and change of 
potential energy $(U)$ as function of displacement for a bistable mechanism, and for (b) a metastable mechanism.

The outline of the rest of this article is as follows: We introduce a PXCM design and analyze its mechanical behavior in Section 2. The experimental validation of the PXCM design is presented in Section 3, followed by an analysis of the energy dissipation characteristics of PXCMs in Section 4. Some concluding remarks and potential future applications are given in Section 5.

\section{PHASE TRANSFORMING CELLULAR MATERIAL (PXCM)}

In this paper we focus our analysis on the PXCM shown in Fig. 2(a), whose unit cell is formed by two sinusoidal beams with the shape given by: $y=(A / 2)[1-\sin (2 \pi(x-\lambda / 4) / \lambda)]$, thickness $t$, depth $b$, wavelength $\lambda$ and amplitude (peak to valley) $A$ that are connected by stiffening walls of thickness $1.5 t$. One half of the symmetric unit cell is shown in Fig. 2(b). Each sinusoidal beam acts as a compliant bistable/metastable mechanism while the stiffening walls provide local support to prevent transverse displacement at the ends of the sinusoidal beams. A characteristic stress $(\sigma=F /(w \cdot b))-\operatorname{strain}(\varepsilon=\delta / L)$ response for a PXCM specimen is shown in Fig. 2(c) in dash-dotted line, where $F$ is the total force applied on the specimen and $\delta$ is the corresponding displacement of the specimen. The PXCM exhibits a linear stress-strain response until the first row of sinusoidal beams collapses at a peak stress, and switches to the other stable configuration; this corresponds to a phase transformation for this row. The subsequent collapse of each row of sinusoidal beams leads to a serrated stress-strain path that is characterized by an average plateau stress $\sigma_{p l}[14]-[16]$. The progressive collapse nature of this process, as shown in Fig. 2(d), is due to a distribution of small imperfections that trigger the rows at slightly different values of (but close to) $\sigma_{\max }$. A similar behavior occurs under unloading, but now the initiation of phase transformation occurs at $\sigma_{\min }$. $\sigma_{\min }$ is positive for a unit cell with a metastable mechanism and is negative for a unit cell with a bistable mechanism. The serrated behavior is caused by the softening of the material response, when one row of cells is undergoing phase transformation, and the subsequent hardening, once the phase transformation is completed. It should be mentioned that, while the PXCM shown in Fig. 2(a) is a $2 \mathrm{D}$ cellular material, phase transformations takes place only for loads in the $y$-direction. We regard this as a 1D PXCM material and the current investigation is focused on the mechanical behavior in the $y$ direction. 


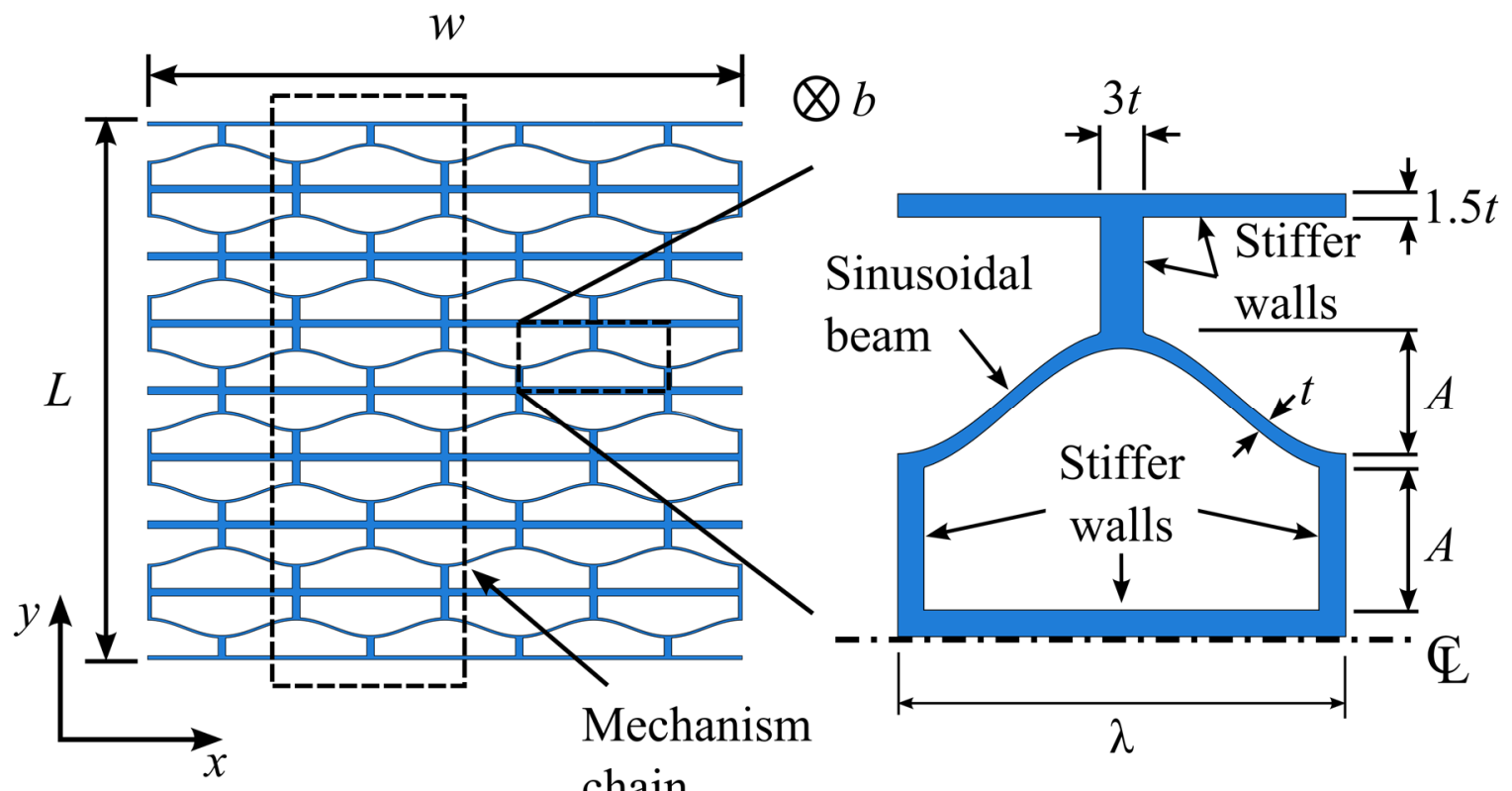

(a)

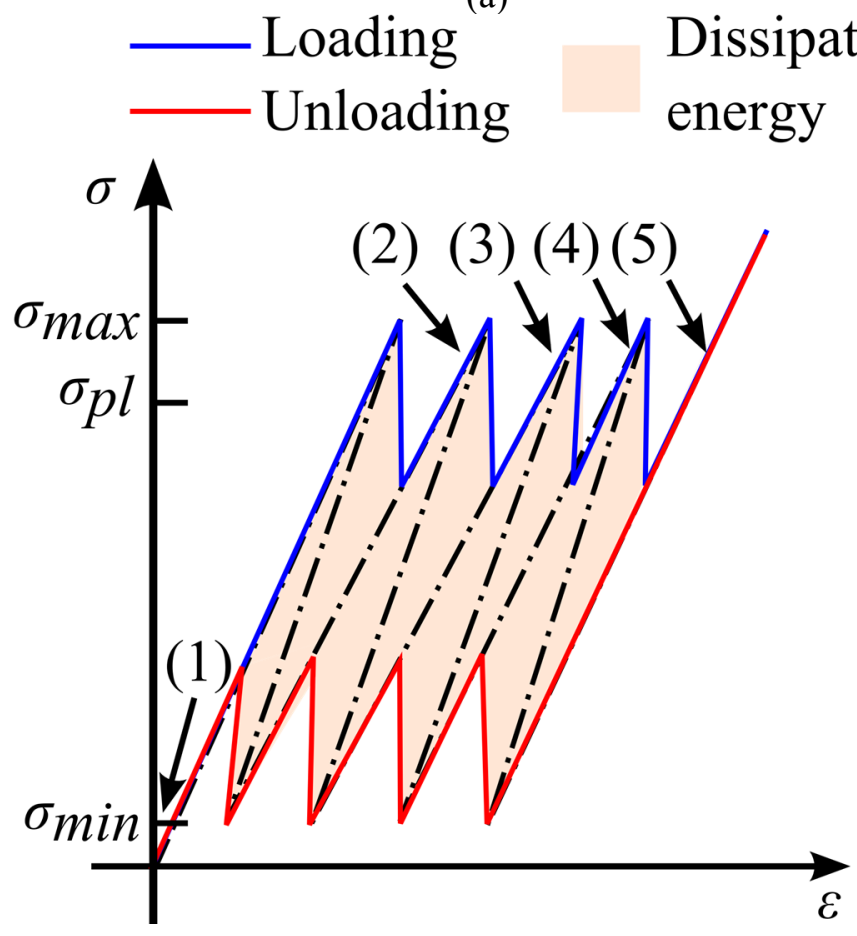

(c) (b)
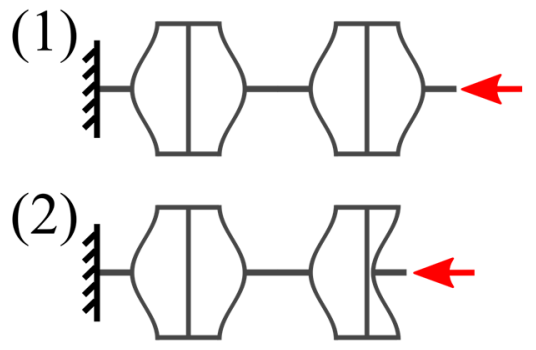

(3)

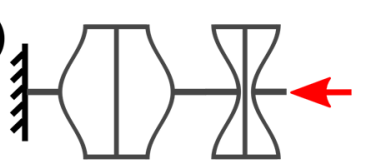

(4)
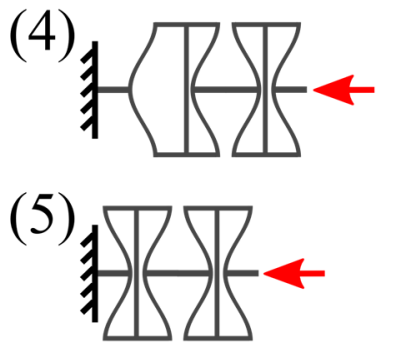

(d)

Fig 2. (a) A design for a phase transforming cellular material. It comprises multiple chains in parallel. Each chain contains multiple unit cells connected in series. (b) Geometry of one half of a symmetric unit cell. (c) Schematic representation of the mechanical behavior of this material under displacement control. (d) Schematic representation of the mechanism configurations at salient points in its mechanical response. 
For the PXCM design presented in this paper, we employ only one base material and postulate that the unit cell can be tailored to exhibit either bistable or metastable behavior by changing its characteristic dimensions. Considering $\pi_{1}=t / \lambda, \pi_{2}=A / \lambda$ and $\pi_{3}=b / t$ as the non-dimensional design parameters, the mechanical behavior of a single sinusoidal beam subjected to a vertical displacement applied at the center can be approximated by using modal superposition of the buckling modes of a straight clamped-clamped beam [17]-[19]. The complete behavior of the sinusoidal beam can be approximated by neglecting the higher modes for $Q=\pi_{2} / \pi_{1}<3$, resulting in the polynomial expression [19]:

$$
F=(1 / 24)(d / A) \pi^{4}\left(6 Q^{2}+4+3 Q^{2}(d / A)^{2}-9(d / A) Q^{2}\right)\left(E \pi_{2} \pi_{3} \lambda^{2} \pi_{1}^{4}\right)
$$

where $d$ is the imposed displacement and $F$ is the corresponding reaction. While the $F$ - $d$ response must be solved numerically for larger values of $Q$, an approximation can be obtained considering a piecewise function that defines each of the characteristic regimes of bistable/metastable mechanism. The $F-d$ response can be characterized by three linear segments (following the trend shown in Fig. 1), which includes an initial linear response with positive slope until $\left(d_{I}, F_{I}\right)$, a second linear response with negative slope until $\left(d_{I I}, F_{I I}\right)$, and a third linear response with a positive slope different to the one of Regime I. This is described by the general equation:

$$
F=k_{i} \cdot d+c_{i}
$$

where $k_{i}=k_{I}, k_{I I}, k_{I I I}$ and $c_{i}=c_{I}, c_{I I}, c_{I I I}$ are the coefficients of the three linear segments passing through the two limit points, $\left(d_{I}, F_{I}\right),\left(d_{I I}, F_{I I}\right)$, and the additional point $\left(d_{I I}, 0\right)$ shown in Fig. 1. These three points are given by:

$$
\begin{aligned}
& F_{I}=\left(10.644 Q^{3}-161.75 Q^{2}+858.42 Q-873.1\right) \frac{E \pi_{2} \pi_{3} \lambda^{2} \pi_{1}^{4}}{12} \\
& d_{I}=\left(-0.0052068 Q^{3}+0.1018 Q^{2}-0.65884 Q+1.6818\right) \pi_{2} \lambda \\
& F_{I I}=\left(-7.0681 Q^{3}+112.86 Q^{2}-620.28 Q+873.1\right) \frac{E \pi_{2} \pi_{3} \lambda^{2} \pi_{1}^{4}}{12} \\
& d_{I I}=\left(0.0098994 Q^{3}-0.1619 Q^{2}+0.91087 Q+0.07253\right) \pi_{2} \lambda \\
& d_{I I I}=\left(-0.017805 Q^{2}+0.20109 Q+1.3818\right) \pi_{2} \lambda
\end{aligned}
$$

for $3 \leq Q<6$, and 


$$
\begin{aligned}
& d_{I}=0.16 \pi_{2} \lambda, F_{I}=8 \pi \frac{E \pi_{2} \pi_{3} \lambda^{2} \pi_{1}^{4}}{12} \\
& d_{I I}=1.92 \pi_{2} \lambda, F_{I I}=-4 \pi \frac{E \pi_{2} \pi_{3} \lambda^{2} \pi_{1}^{4}}{12} \\
& d_{I I I}=1.99 \pi_{2} \lambda
\end{aligned}
$$

for $Q \geq 6$. According to these approximations, the sinusoidal beams exhibit one stable and one metastable configuration when $2.31 \leq Q \leq 2.41$ and, two stable configurations when $Q>2.41$. There is only one stable configuration that corresponds to the as-fabricated shape of the sinusoidal beam when $Q<2.31$. The maximum strain in the base material of the sinusoidal beam during the deflection is given by $\varepsilon_{m x}=2 \pi^{2} \pi_{1} \pi_{2}$. We use this quantity as a design parameter to ensure the reversibility of the phase transformation by restricting $\varepsilon_{\max }<\varepsilon_{y}$, where $\varepsilon_{y}=\sigma_{y} / E$ is the yield strain (and $\sigma_{y}$ is the yield stress) of the base material.

As shown in Fig. 2(a), a PXCM specimen is composed of an array of $N_{c}=w / \lambda$ mechanism chains, that contains $N$ sinusoidal beams connected in series. Assuming that just one complete row of sinusoidal beams is transitioning to the second stable configuration at a time, and that the system is over damped, we can relate the behavior of a single sinusoidal beam with the global mechanical behavior of the PXCM specimen. It is worth mentioning that chains of bistable mechanisms have been previously employed to analyze the behavior of materials that present phase transformation at the atomic level and structures whose behavior resemble phase transformations [5], [6], [20]-[23]. We build on these existing models to characterize the behavior of the PXCM. Defining $n_{I}, n_{I I}$ and $n_{I I I}$ as the number of sinusoidal beams per chain that are in regimes I, II and III respectively (see Fig. 1(a)-(b)), the global strain as a function of the applied stress in the PXCM specimen can be obtained from

$$
\varepsilon(\sigma)=\left(n_{I} \varepsilon_{I}(\sigma)+n_{I I} \varepsilon_{I I}(\sigma)+n_{I I I} \varepsilon_{I I I}(\sigma)\right) / N \text { With } \sigma_{\min } \leq \sigma \leq \sigma_{\max } \text { and } n_{I}+n_{I I}+n_{I I I}=N
$$

Where $\varepsilon_{i}(\sigma)$ corresponds to the local strain for a row of sinusoidal beams in a particular regime measured with respect to its undeformed configuration and can be derived from Eqs. 1-2. As shown in Eq. 3 the stress-strain response of the PXCM specimen depends on $N$. Considering $k_{I}, k_{I I}$ and $k_{I I I}$ as the effective stiffness of a sinusoidal beam that is in regimes I, II and III respectively, the effective stiffness of the PXCM is given by $k=\left(\left(n_{I} / k_{I}\right)+\left(n_{I I} / k_{I I}\right)+\left(n_{I I I} / k_{I I I}\right)\right)^{-1}$. This means that adding sinusoidal beams to the chain decreases the effective stiffness of the PXCM. Under the assumption that only one active row of sinusoidal beams is undergoing transition (as shown in Fig. 2(d)), the effective stiffness of 
the portion of the PXCM that contains sinusoidal beams in regimes I and III decreases with $N$ while the stiffness of the active row remains constant $k_{I I}$. Once $N$ reaches or exceeds a critical value, the following condition is met:

$$
\left(\frac{n_{I}}{k_{I}}+\frac{n_{I I I}}{k_{I I I}}\right)^{-1} \leq\left|k_{I I}\right| .
$$

The PXCM specimen that satisfies this equation will exhibit a snapback behavior (i.e. a reversal in the displacement along with a reduction in the equilibrium force needed to drive the transformation) when undergoing a change of phase. A schematic of the response of a PXCM that exhibits snapback behavior is depicted in Fig. 2(c). The black dash-dot line represents the stress-strain curve per Eq. 3 for a material that exhibits snapback. The red and blue solid lines respectively represent the displacement-controlled loading and unloading behavior of the material. Note that the displacement controlled responses do not reflect the reversal in displacement associated with the snapback because most simple path following algorithms lack the capability to address mechanical responses that have segments where both the force and displacement can be multi-valued. Once the row of sinusoidal beams undergoing a change in configuration reaches a limit point ( $\sigma_{\max }$ for loading and $\sigma_{\min }$ for unloading) a further increase (or decrease for unloading) in the displacement, under displacement control conditions, causes these sinusoidal beams to snapthrough to the other stable configuration. This snapthrough at the unit cell level allows the material to present hysteresis upon loading and unloading. Non-equilibrium transitions such as snapthroughs or snapbacks at the unit cell level cause a local release of strain energy. This energy gets dissipated by the damping of the base material and/or by the interaction of the sinusoidal beams with the medium (e.g., air or any other fluid). As can be observed in Fig. 2(c), the energy dissipated is denoted by the area enclosed by the hysteresis curve. This results in dissipation of energy at the cellular material level even when the base materials exhibit little or no dissipation. While the path of the hysteretic cycle shown in Fig. 2(c) is calculated for a base material with non-zero intrinsic dissipation, a detailed dynamic analysis of the damping effect for base materials with a wide range of intrinsic dissipation is included in Section 4.1

To study the effect of $N$ on the behavior of the PXCM specimen, we report the variation of $\sigma_{p l} / \sigma_{\max }$ as a function of $N$ for a range of $Q$ between 2.31 and 6 in Fig. 3(a). We note three distinct regions in this figure: R-1, R-2 and R-3 (see inset in Fig. 3(a)). Region R-1 is characterized by a constant value of $\sigma_{p l} / \sigma_{\max }$, and it corresponds to low values of $N$ for which there is no snapback (i.e., Eq. 4 is not satisfied). Region R-2 starts with a sharp kink in the curve. In R-2 the material begins to exhibit snapback leading to a rise in $\sigma_{p l} / \sigma_{\max }$ until it saturates with an asymptotic value of 1 . Region R-3 is described by 
this asymptotic value and corresponds to a regime where the behavior of the PXCM is independent of $N$. We also observe from Fig. 3(a) that $Q$ has no effect on the $\sigma_{p l} / \sigma_{\max }-N$ curves for $Q \geq 6$.

The behavior of the PXCM in each of the three regions can be explored by studying its stress-strain response for a complete loading and unloading cycle under displacement control. We pick a specific PXCM design characterized by $\pi_{1}=0.012, \pi_{2}=0.072(Q=6)$ and consider specimens with $N=4, N=$ 14 and $N=10000$ for illustration. All these specimens have the following limit stress values: $\sigma_{\max }=8.08$ $\mathrm{MPa}$ and $\sigma_{\min }=-4.04 \mathrm{MPa}$. Figure 3(b) shows the behavior of the PXCM specimen with $N=4$, which corresponds to the region R-1 described in Fig. 3(a). In this case, Eq. 4 is not satisfied and no snapback is observed. As a result, both loading and unloading curves coincide for this particular case and there is no dissipation associated with the phase transformation when the specimen is loaded under displacement control conditions. The specimen with $N=14$ falls into region R-2 (Fig. 3c). In this case the inequality in Eq. 4 is satisfied and the specimen exhibits snapback behavior. The snapback makes the PXCM specimen exhibit finite energy dissipation under displacement control. The energy dissipated per cycle is denoted by the area enclosed by the hysteresis curve. Fig. 3(d) depicts a PXCM specimen with $N=10000$ which falls into the region R-3. We note that the snapback during phase transformation becomes more prominent as $N$ increases, leading to a reduction of the amplitude of the serration (i.e. $\frac{\sigma_{p l}}{\sigma_{\max }} \rightarrow 1$ ) and a corresponding increase in the energy dissipated per load-unload cycle. 

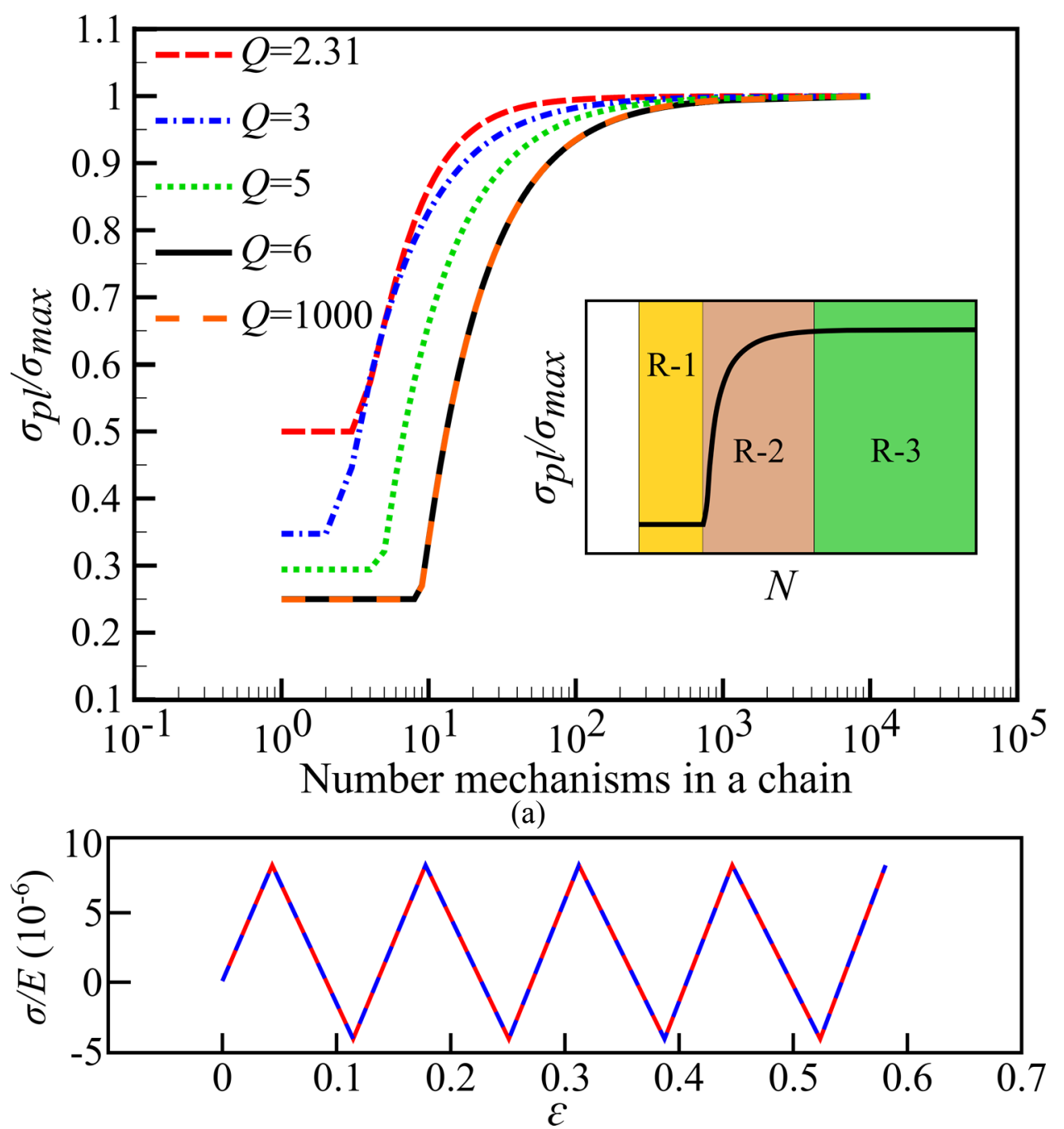

(b)

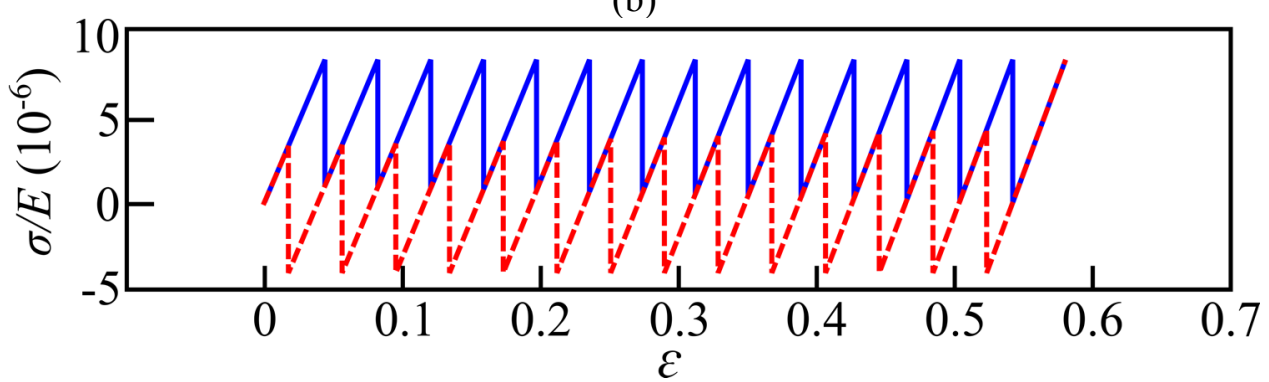

(c)

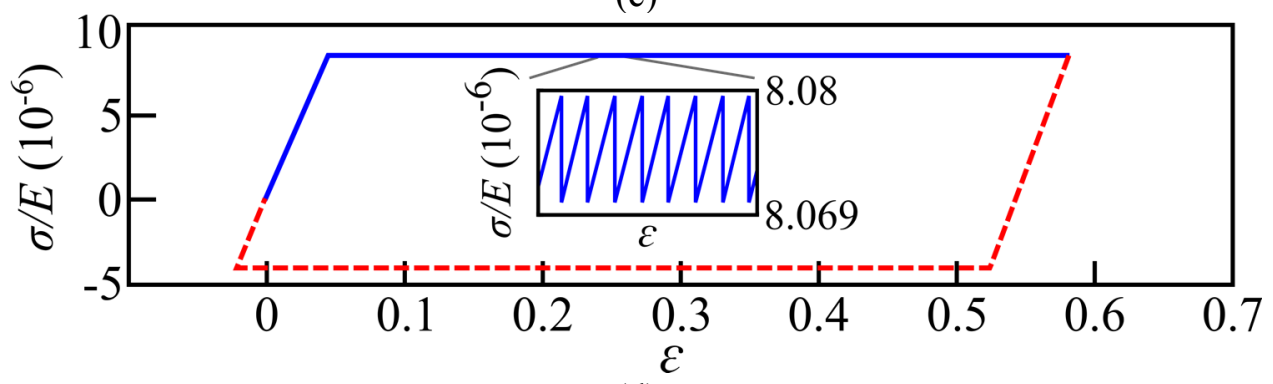

(d) 
Fig. 3. (a) $\sigma_{p l} / \sigma_{\max }$ as a function of $N$ for different values of $Q$. (Inset: characteristic $\sigma_{p l} / \sigma_{\max }$ vs $N$ curve exhibiting three regions: R-1, R-2 and R-3). The stress-strain responses of specific materials from the three regions with $Q=6$ are shown as: (b) R-1: $N=4$, (c) R-2: $N=14$ and (d) R-3: $N=10000$. The solid blue line represents the loading path and the red dashed line the unloading path.

\section{EXPERIMENTAL VALIDATION}

We fabricated a specimen of the PXCM shown schematically in Fig. 2(a) using an Object Connex500 3D printer with a photo-cured polymer (RGD $8530, E=1 \mathrm{GPa}$ and $\sigma_{y}=19 \mathrm{MPa}$ ) as a base material. The geometry of the unit cell considered is given by $\pi_{1}=0.012, \pi_{2}=0.084$ (i.e., $Q=7$ ), $\pi_{3}=27.78$ and $\lambda=60 \mathrm{~mm}$. The specimen has 4 chains with each chain having $N=24$ sinusoidal beams. This material was tested in a universal test machine for loading and unloading (MTS Insight 10 equipped with a $10 \mathrm{kN}$ load cell MTS 661.19F-02). Figure 4 shows the behavior of the tested PXCM specimen (solid red line) compared with the response predicted by Eq. 3 considering displacement control (solid blue line) and the snapback path (dashed line). An important observation from this figure is that the PXCM tested exhibits a long, albeit serrated, plateau which makes these type of materials attractive for energy absorption applications [24].

The increase in the stress for $\varepsilon>0.6$ can be attributed in part to nonlinearities in the material response and intrinsic stochastic variations of the fabrication process. These variations lead to a progressive transformation beginning with the weaker cells (low $\sigma_{\max }$ ) and ending with the stronger ones (high $\sigma_{\max }$ ). Fig. 5 shows snapshots of the deformation of the PXCM during the compressive part of the testing. The tests validated our assumption that just one row of unit cells is changing phase at an instant in time. As an additional validation step, we use the images to measure permanent deformation. As such, the shape of 20 sinusoidal beams was discretized using 15 nodes equally distributed through each beam. The position of these nodes was measured before and after several loading and unloading cycles using photogrammetry and we found that the average change of position of each of the nodes compared with its configuration after the test is $0.4 \pm 0.02 \%$ allowing us to conclude that the material recovered its initial shape after the completion of the test. 


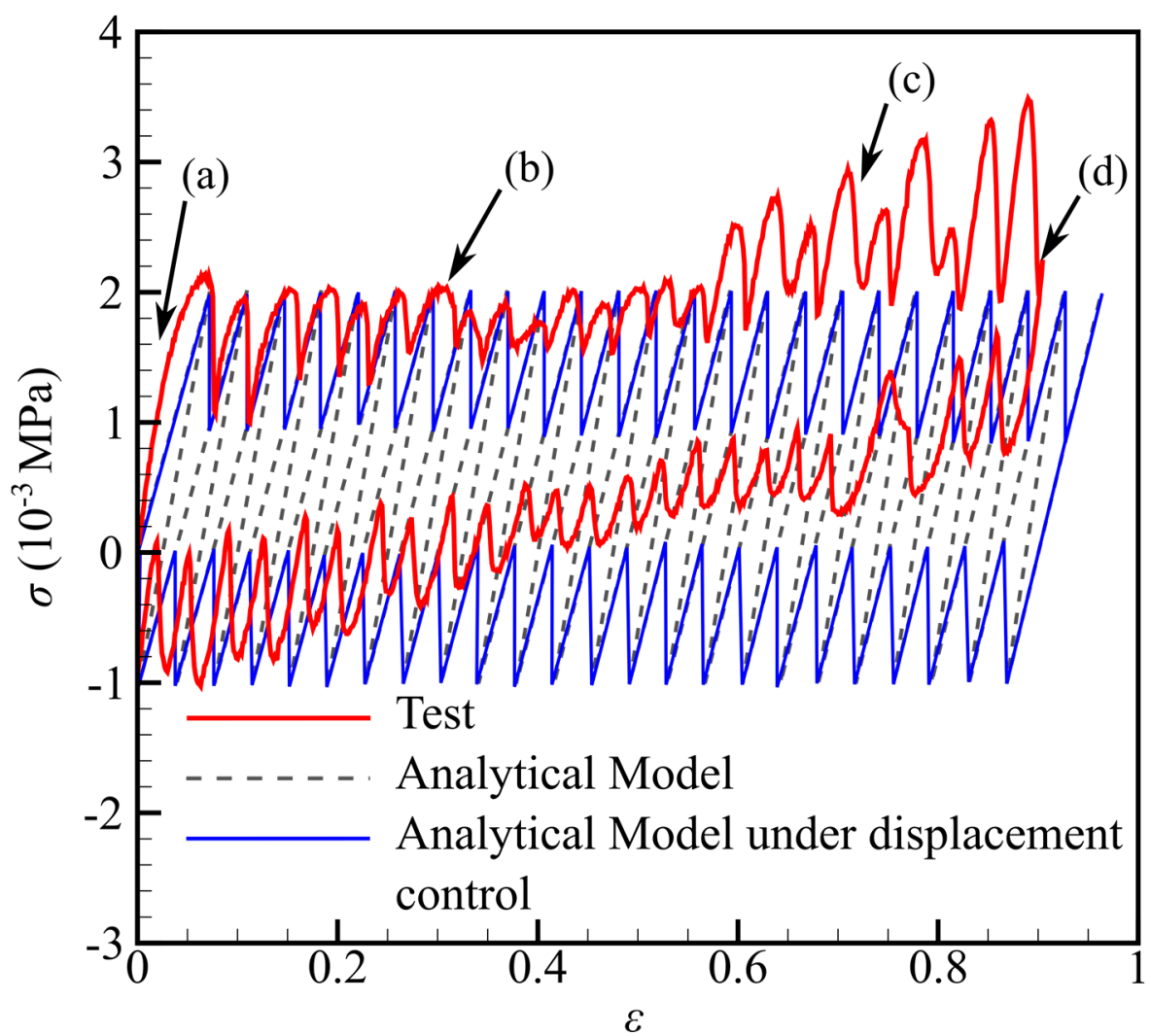

Fig. 4. Stress-strain behavior of fabricated PXCM material compared with the behavior predicted by the analytical model for a compression/tension test. The labels (a)-(d) indicate the snapshots shown in Fig. 5.

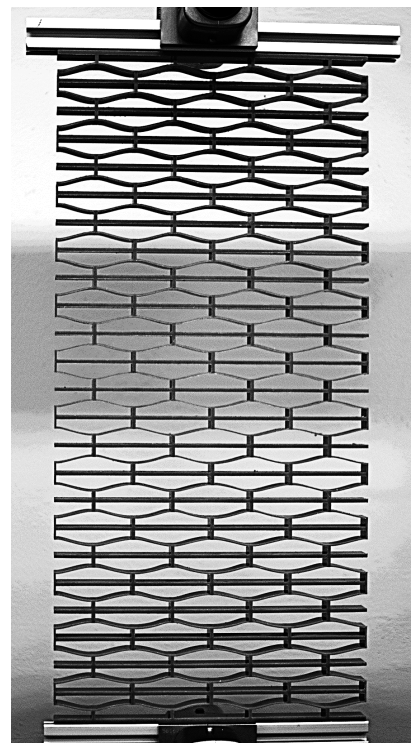

(a)

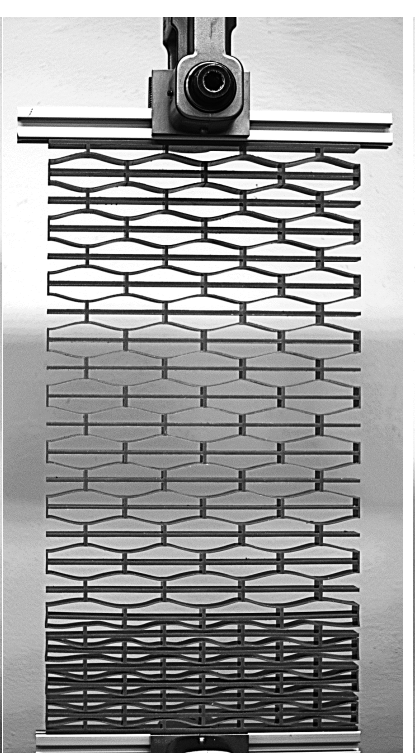

(b)

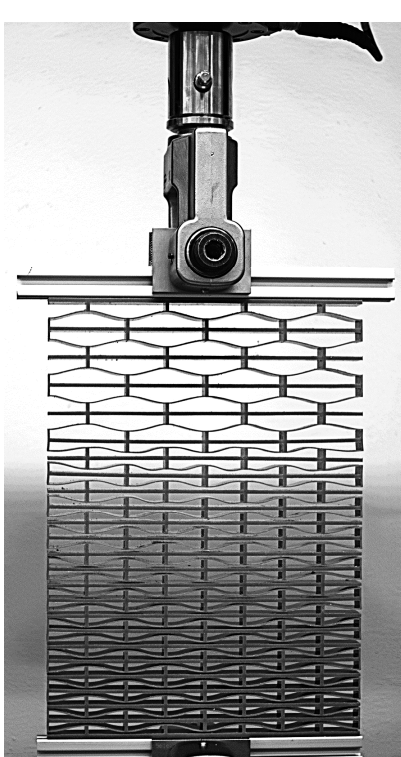

(c)

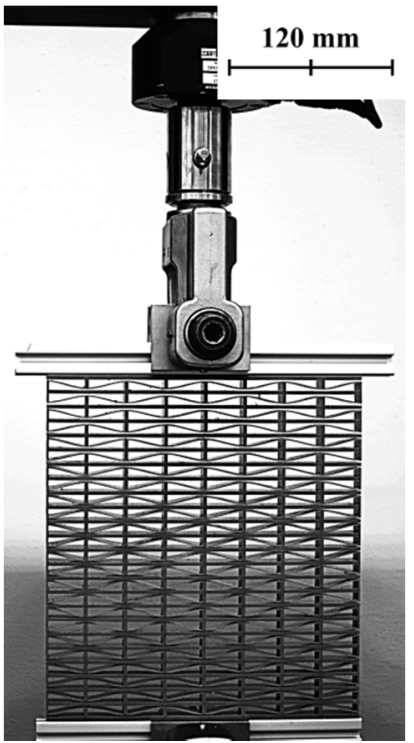

(d)

Fig. 5. Deformation of phase transforming cellular material at different strains subjected to compression. (a) $\varepsilon=0$, (b) $\varepsilon=0.3$, (c) $\varepsilon=0.7$ (d) $\varepsilon=0.87$ 


\section{ENERGY DISSIPATION}

\subsection{Cycling loading}

As discussed in Section 2, PXCMs are able to dissipate energy due to their hysteretic behavior. For a given unit cell topology and geometry, the energy dissipation exhibited by a PXCM specimen has a nonlinear dependence on the number of unit cells included in such specimen. We employ the loss factor defined as $\eta=W_{d} /\left(2 \pi W_{i}\right)$ as a measure of the ability of a material to dissipate energy [24]-[26]. For this definition of $\eta, W_{i}$ is the work done in deforming a PXCM up to a given maximum applied strain and $W_{d}$ is the energy dissipated in a complete loading-unloading cycle. Fig. 6 shows the variation of $\eta$ as a function of $N$ for various values of values of $Q$ between 2.31 and 6 . The stress-strain responses for the various specimens are computed using the equations $1-3$ following the same procedure describe in Sect. 2. The specimens are loaded until their respective densification strains $\left(\varepsilon_{d}=1-1.4 \rho_{r}\right.$ where $\rho_{r}$ corresponds to the relative density) are reached [27]-[29]. The inset in Fig. 6 shows that all the curves in the figure exhibit three distinct regions: R-1, R-2 and R-3 similar to those observed in fig. 3(a). Each of these regions is also linked to the capacity of the PXCM specimen to exhibit snapback. Region R-1 is characterized by a value of loss factor equal to zero and corresponds to low values of $N$ for which there is no snapback. As shown in Fig. 3(b), the progressive collapse of the bistable/metastable mechanisms that form the unit cell of the PXCM is not a sufficient condition to obtain energy dissipation when the material is loaded under displacement control. In order to dissipate energy under displacement control, $N$ should be equal to or larger than the critical value given by Eq. 4, to induce snapback behavior in the PXCM. The start of region R-2 is marked by a sharp rise in $\eta$. This occurs when there is a sufficient number of mechanisms in the specimen for the material response to exhibit a snapback (according to Eq. 4) and a separation between the loading and the unloading paths starts to occur as shown in Fig. 3(c). We observe that $\eta$ increases as a function of $N$ until it reaches an asymptotic value for sufficiently large values of $N$. Region R-3 is described by this asymptotic value of $\eta$ and corresponds to a regime where the dissipation performance of a PXCM specimen is independent of $N$. The representative mechanical response of a material in region R-3 is shown in Fig. 3(d). We also observe that $Q$ has an effect on $\eta-N$ curves. However, all the curves remain identical once $Q \geq 6$. This trend is similar to what was observed in Fig. 3(a). 


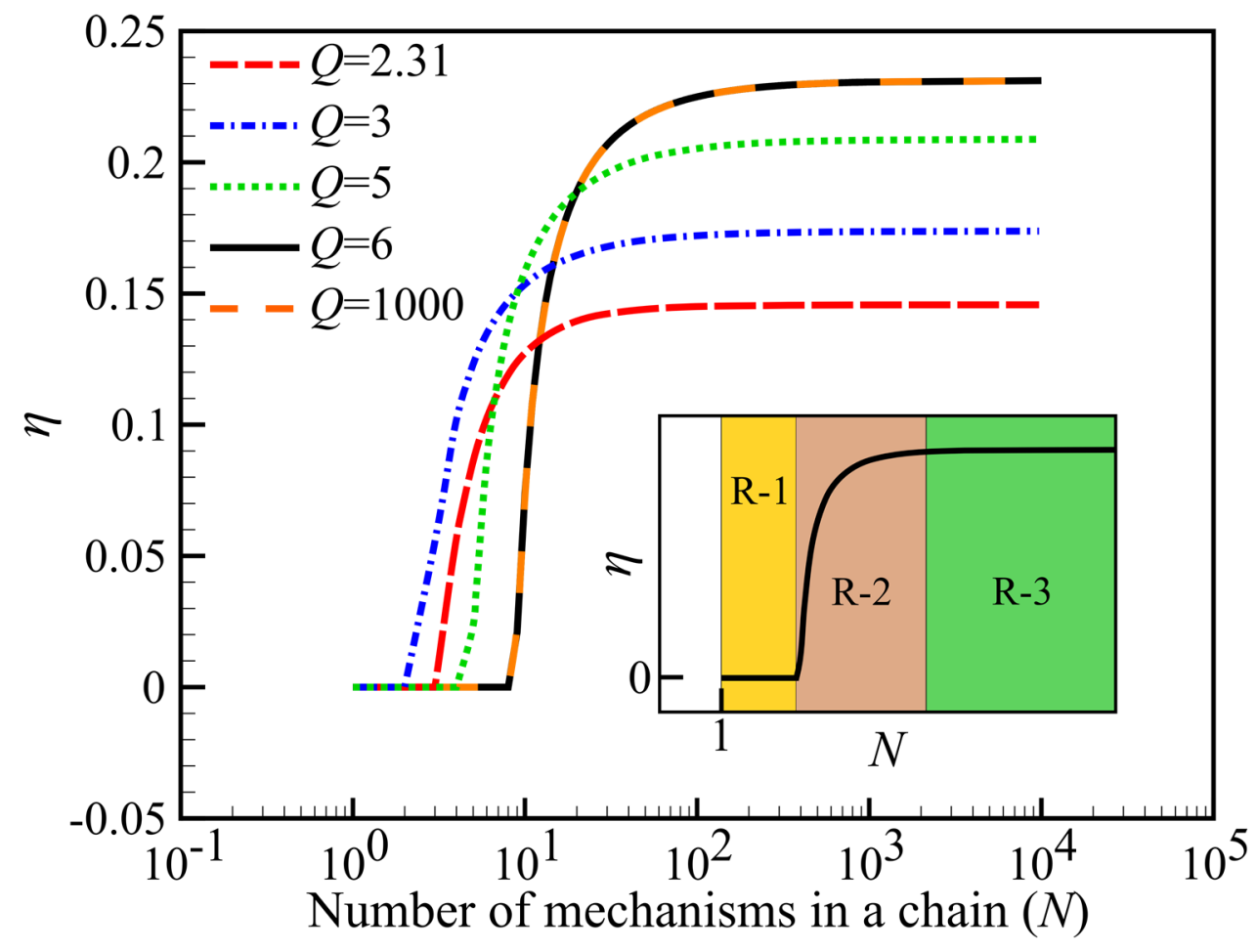

Fig. 6. Dependence of $\eta$ on $N$. After $N=1000$ a plateau starts to form for any value of $Q$. The inset figure shows a characteristic $N-v s-\eta$ response that is partitioned into three distinct regions.

In order to understand the effect of the intrinsic dissipation of the base material on the loss factor of the PXCM, we considered a simplified dynamic model that consists of a spring, a dashpot, a mass and a bistable/metastable system (see inset Fig. 7) that is undergoing phase transformation. This model mimics the mechanical response of a large PXCM specimen (i.e. one which belongs to R-3 in Figs. 3(a) and 6) subjected to a loading and unloading cycle under quasi-static displacement control with an amplitude that is large enough to produce the change of configuration of a single row of mechanisms through snapthrough. This allows us to determine a more accurate value of $\eta$ that takes into consideration the damping and inertia of the base material. The model assumes that the mass of the sinusoidal beams undergoing a change in configuration is concentrated at a single point with mass $m$ connected to (i) a spring that represents the stiffness of the overall material ( $k_{\text {eff }}$ ), (ii) a dashpot that represents the intrinsic damping of the base material $\left(\eta_{\text {base }}\right)$, and (iii) a bistable/metastable system $\left(B_{n}\right)$ that represents the forcedisplacement behavior of the bistable/metastable mechanism, which is described by Eqs. 1 and 2 (see Fig. 1). Since the PXCM is in region R-3 and Eq. 4 is satisfied (i.e., $k_{\text {eff }}<\left|k_{I I}\right|$ ), the snapthrough in $B_{n}$ causes a local release of strain energy that is converted into kinetic energy. This manifests as vibratory motion of the neighboring parts of the specimen. The rest of the PXCM that is not undergoing phase transition has a mass that is significantly larger phase than $m$ and is assumed to be at rest. As such, the vibration is 
confined to the sinusoidal beams of the row undergoing transformation. The values of $\eta_{\text {base }}$ considered on this study correspond to the loss factors measured in accordance with ASTM E756-05 $[25]$. We also consider that the PXCM operates in vacuum in order to eliminate the contribution of viscous damping due to the interaction with the surrounding air. We recognize that the overall dimensions and density of the base material of the unit cell affect the inertia of the system and account for these effects by considering different value of mass $m$ while keeping $Q$ and $k_{\text {eff }}$ constant.

Fig.7 shows the variation of the loss factor $\eta$ for a PXCM specimen as a function of $\eta_{\text {base }}$ for a specimen with $Q=5.4307$ and $N=10000$. For this value of $Q, k_{I}=k_{I I I}$, and hence $k_{\text {eff }}=k_{I} /(N-1)$. In this analysis, $k_{\text {eff }}=7643.2 \mathrm{~N} / \mathrm{m}$ and $\eta_{\text {base }}$ is varied in the interval $\left[10^{-4}-10^{0}\right]$ to account for typical engineering materials (i.e. steel, aluminum, polymers at $30^{\circ} \mathrm{C}[30]$ ). Specifically PXCMs made out of polymer, aluminum and steel are highlighted. These designs correspond to $\pi_{1}=[0.0071-0.02]$, $\pi_{1}=[0.0028-0.0029]$ and $\pi_{1}=[0.002-0.0021]$ respectively. Also, different values of $m=1,10$ and $100 \mathrm{~g}$ were considered to account for the associated length scale of different units cells that form the PXCM. As observed in Fig. 7, the system reaches a plateau at $\eta=0.22$ for large values of $\eta_{\text {base }}$. This value is the same as the one predicted by Eqs. 1-3 (see fig. 6) for large specimen (e.g. one that belongs to the region R-3) of an over damped PXCM. For lower values of $\eta_{\text {base }}$, the unstable nature of the snapback during phase transformation causes the mass to vibrate. The amplitude of this vibration is a function of the potential energy stored up to the traversal point and the inertia provided by $m$. Depending on the amplitude of the vibration, the bistable/metastable mechanism may be driven back to its original configuration by inertia forces, which leads to a reduction in $\eta$. While these uncontrolled fluctuations are independent of the applied displacement, they depend on the mass. Evidently, a further increase in with $m$ leads to further reduction in $\eta$ for a given material. However, miniaturizing PXCMs with values of $\lambda$ in the micron-scale (e.g., using microfabrication) will decrease the mass of the sinusoidal beam. Ultimately, this leads to an increase of the viscous forces over inertia forces ratio resulting in an improvement of $\eta$. Another important observation from Fig. 7 is that for low values of $\eta_{\text {base }}$ (e.g. the case of steels $\eta_{\text {base }}=\left[1.2 \times 10^{-4}-1.42 \times 10^{-4}\right]$ and Al alloys $\left.\eta_{\text {base }}=\left[2.05 \times 10^{-4}-3.22 \times 10^{-4}\right]\right)$, a PXCM may produce an amplification of an $\eta$ that is $100-1000$ times greater than that of the base material. In contrast, commercially available metal foams only produce a amplification factors of 5-10 times[24]. Our analysis underestimates the amplification of $\eta$ since our simplified system does not consider any interactions

\footnotetext{
${ }^{1}$ This is calculated using the first mode of a cantilever beam which assumes that the damping measurements remains in the linear range and the material behaves in accordance to linear viscoelastic theory.
} 
with the medium that surrounds the PXCM specimen or displacement constraints on $m$ due to the geometrical periodicity of the material. Since the vibration is confined to the sinusoidal beam of the row undergoing transformation, the models does not consider any transfer of kinetic energy to the rest of the specimen. Both of these may help to reduce the amplitude of the vibrations resulting from the phase transition and thus increase the predicted $\eta$. It is also worth mentioning that this model considers quasistatic loading of the PXCM specimen. Any lossless (e.g. perfectly elastic) homogeneous solid material tested under the same conditions will result in a loss factor of zero. However, the estimated loss factor for a PXCM made of such a lossless material can be as high as $\eta=0.23$ (as shown in Fig. 6) for the PXCM design presented here.

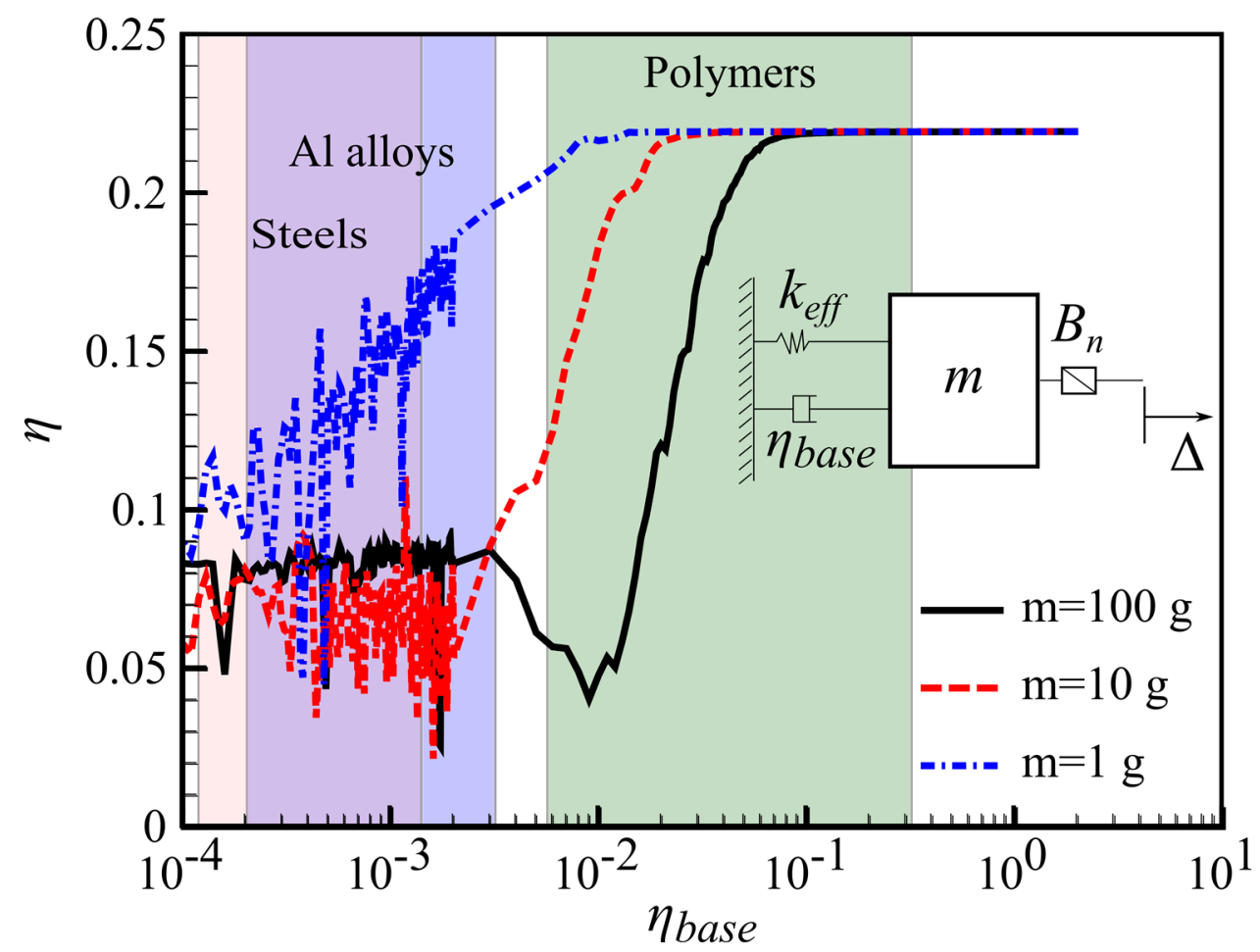

Fig. 7. Effect of $\eta$ as a function of $\eta_{\text {base }}$ for a material described by $Q=5.4307, N=10000$ and $k_{\text {eff }}=7643.2 \mathrm{~N} / \mathrm{m}$. PXCMs made out of polymer, aluminum and steel consider $\pi_{1}=[0.0071-0.02]$, $\pi_{1}=[0.0028-0.0029]$ and $\pi_{1}=[0.002-0.0021]$, respectively. Values of loss factor for the PXCM are calculated using a simplified spring-dashpot-mass-bistable/metastable system under quasi-static displacement control. In contrast, any non-PXCM material would give $\eta=0$ under the same conditions. Values of $\eta_{\text {base }}$ are obtained from[30].

\subsection{In-plane crushing}

An important application of cellular materials is in the packing and protection of goods, persons and structures due to its capacity of absorb energy and mitigate a load by extending its duration [31]-[33]. 
Due to their long constant plateau in stress, PXCM may be attractive for these applications. In Fig. 8(a)(b) we compare the theoretical energy absorbed per unit mass of a PXCM with the corresponding values for the hexagonal and kagome honeycombs made out of metals and polymers respectively. These charts are constructed by plotting the data for a large number of geometries of each of the three materials, generating the convex hulls of the data points for each material and shading the bounded region.

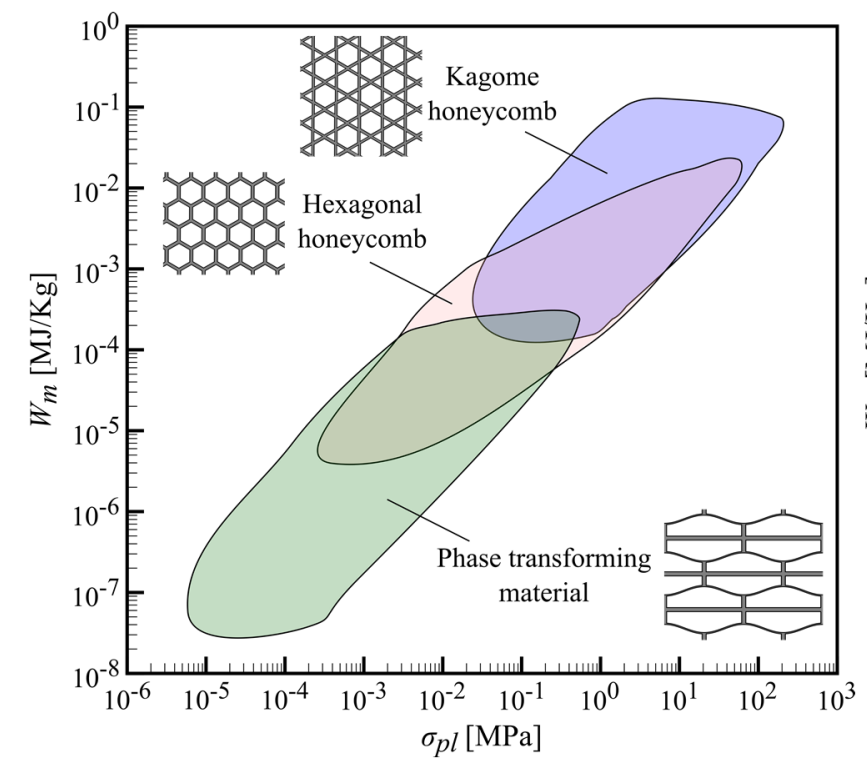

(a)

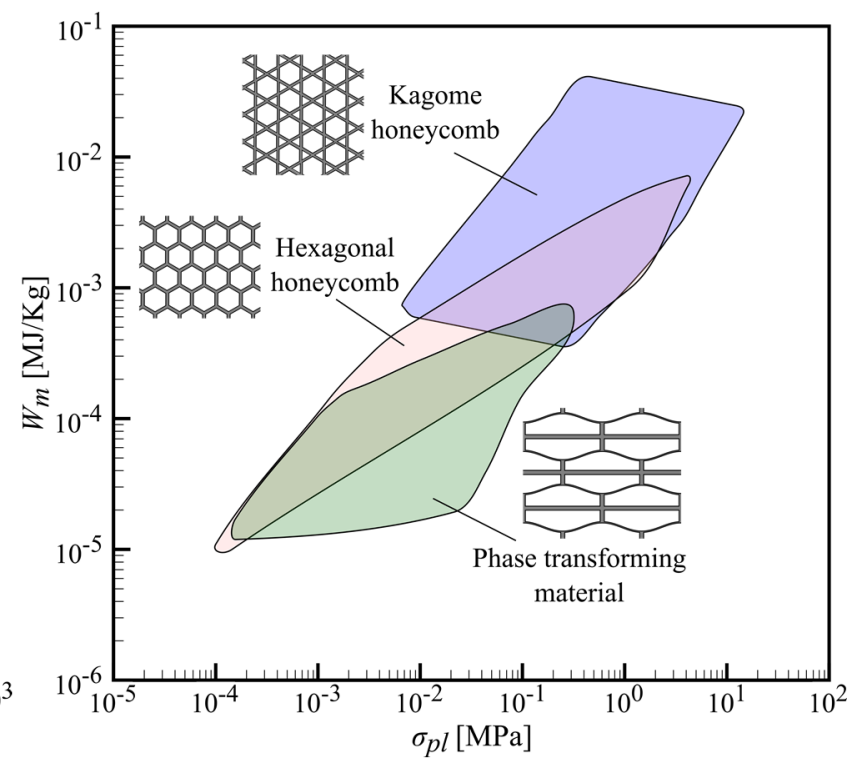

(b)

Fig. 8. Comparison of energy dissipation of the phase transforming cellular material with kagome and hexagonal honeycombs. (a) Considering metals as base material: $E=[12.5-220] \mathrm{GPa}$, $\sigma_{y}=[8-1245] \mathrm{MPa} \rho=[1.74-11.4] \mathrm{Mg} / \mathrm{m}^{3}$. (b) Considering polymers as base material: $E=[0.5-5] \mathrm{GPa}, \sigma_{y}=[8.3-95] \mathrm{MPa}$ and $\rho=[0.89-2.2] \mathrm{Mg} / \mathrm{m}^{3}$.

The energy dissipated by the PXCM is calculated using Eq. 3 considering a sufficiently large material to assume that the PXCM is R-3 of Figs. 3(a) and 6 (i.e. $N \rightarrow \infty$ ). On the other hand, the energy dissipated by the hexagonal and kagome honeycombs is approximated by the multiplication of plastic stress plateau also (labeled $\sigma_{p l}$ for simplicity) and the densification strain $\left(\varepsilon_{d}\right)[24],[34] . \sigma_{p l}$ for the hexagonal honeycomb and the kagome on its maximum direction is calculated as $\sigma_{p l}=0.5 \rho_{r}$ where $\rho_{r}$ corresponds to the relative density of each honeycomb [27]-[29]. We observe that the performance space for PXCMs overlaps with the corresponding spaces for the hexagonal and kagome honeycombs. In the overlap regions, the energy absorbed per unit mass by the material presented here is comparable to that absorbed by the hexagonal and kagome honeycombs made of the same material at similar $\sigma_{p l}$. However, 
unlike these honeycombs, where the energy absorption is due to plastic deformation, the base material of the PXCM remains in the elastic regime throughout the energy absorption process described in section 2.

\section{CONLCUSIONS}

We introduced a new class of cellular materials, PXCM, that exhibit phase transformation. These materials consist of unit cells that exhibit multiple stable configurations such that each stable configuration is associated with a material phase. Phase transformations correspond to a limit point traversal in the unit cells that causes the cells to switch between stable configurations. The progressive phase transformation of the each unit cell in the PXCM specimen leads to a serrated force-displacement behavior similar to the one presented in the martensitic transformations in shape memory alloys and conformation changes in proteins. The design of the unit cell of the PXCMs to obtain reversible phase transformation properties must meet three fundamental requirements: (i) use of bistable/metastable mechanism to allow the equilibrium conditions between phases, (ii) the unit cell must counteract the Poisson effect to provide the balance in strain energy required to obtain bistable or metastable behavior of the compliant mechanism, and (iii) the geometry of the unit cell must be chosen such that the stress at any point in the cell does not exceed the yield stress of the base material during phase transformation.

We have also demonstrated the concept of PXCM through material design using unit cells that exhibit two stable/metastable configurations. The correlation between experiments and the proposed analytical model suggests that the response of the system and design methodology is robust and can be used to generate other designs for phase transforming cellular material. Future work will focus on extending this concept so that the unit cells can be designed to undergo phase transformation under multiaxial stress condition (e.g., 2D and 3D PXCMs materials), investigate the effect of defects, loading rate under dynamic conditions. While the PXCM material reported in Figs. 4-5 was 3D printed at the $m m$-scale, we surmise that the phase transforming mechanisms proposed in this work can be scalable to other length scales; especially to smaller scales where inertia effects are negligible. As the material is designed to operate in the elastic regime of the base material, we expect the quasi-static response of the tested samples to be representative of PXCMs at other length scales, unless inertia and size effects of the base material come into play.

The extension of the notion of phase transformation to cellular materials allows the increase of versatility of these materials. As an example, the phase transformation can be used for energy dissipation. Additionally, the ability of the material to have multiple stable configurations could enable the use PXCMs with programmable properties where each of the phases of the material offers a different 
mechanical behavior. This concept could be used for wave guiding, filtering and shape morphing, among many others.

\section{ACKNOWLEDGMENTS}

D. R and P.D.Z. gratefully acknowledge the generous financial support of the General Motors Company.

\section{REFERENCES}

[1] G. Krauss, "Steels: heat treatment and processing principles," ASM Int. Mater. Park, 1989.

[2] E. Bayraktar, F. A. Khalid, and C. Levaillant, "Deformation and fracture behaviour of high manganese austenitic steel," J. Mater. Process. Technol., vol. 147, no. 2, pp. 145-154, 2004.

[3] P. D. Zavattieri, V. Savic, L. G. Hector Jr., J. R. Fekete, W. Tong, and Y. Xuan, "Spatio-temporal characteristics of the Portevin-Le Châtelier effect in austenitic steel with twinning induced plasticity," Int. J. Plast., vol. 25, no. 12, pp. 2298-2330, 2009.

[4] T. Duerig, K. Melton, and D. Stöckel, Engineering aspects of shape memory alloys. ButterworthHeinemann, 2013.

[5] G. Puglisi and L. Truskinovsky, "Rate independent hysteresis in a bi-stable chain," J. Mech. Phys. Solids, vol. 50, no. 2, pp. 165-187, 2002.

[6] G. Puglisi and L. Truskinovsky, "Mechanics of a discrete chain with bi-stable elements," J. Mech. Phys. Solids, vol. 48, no. 1, pp. 1-27, 2000.

[7] F. Li, J. Yuan, and C. Mou, "Mechanical unfolding and refolding of proteins: an off-lattice model study," Phys. Rev. E, 2001.

[8] I. Benichou and S. Givli, “The hidden ingenuity in titin structure,” Appl. Phys. Lett., vol. 98, no. 9, p. 091904, 2011.

[9] B. Smith, T. Schäffer, and M. Viani, "Molecular mechanistic origin of the toughness of natural adhesives, fibres and composites," Nature, 1999.

[10] K. Eom, D. Makarov, and G. Rodin, "Theoretical studies of the kinetics of mechanical unfolding of cross-linked polymer chains and their implications for single-molecule pulling experiments," Phys. Rev. E, 2005.

[11] L. L. Howell, Compliant mechanisms. John Wiley \& Sons, 2001.

[12] L. L. Howell and S. S. Rao, "Reliability-Based Optimal Design of a Bistable Compliant Mechanism," vol. 116, no. December, pp. 1115-1121, 1994. 
[13] Z. P. Bazant and L. Cedolin, Stability of Structures: Elastic, Inelastic, Fracture, and Damage Theories. Courier Dover Publications, 1991.

[14] S. D. Papka and S. Kyriakides, "In-plane compressive response and crushing of honeycomb," $J$. Mech. Phys. Solids, vol. 42, no. 10, pp. 1499-1532, 1994.

[15] S. D. Papka and S. Kyriakides, "Experiments and full-scale numerical simulations of in-plane crushing of a honeycomb," Acta Mater., vol. 46, no. 8, pp. 2765-2776, 1998.

[16] S. D. Papka and S. Kyriakides, "In-plane crushing of a polycarbonate honeycomb," Int. J. Solids Struct., vol. 35, no. 3, pp. 239-267, 1998.

[17] M. Vangbo, "An analytical analysis of a compressed bistable buckled beam," Sensors Actuators A Phys., vol. 69, no. 3, pp. 212-216, 1998.

[18] J. Qiu, J. H. Lang, and a. H. Slocum, “A Curved-Beam Bistable Mechanism,” J. Microelectromechanical Syst., vol. 13, no. 2, pp. 137-146, 2004.

[19] J. Qiu, "An electrothermally-actuated bistable MEMS relay for power applications," Massachusetts Institute of Technology, 2003.

[20] I. Benichou, E. Faran, D. Shilo, and S. Givli, "Application of a bi-stable chain model for the analysis of jerky twin boundary motion in NiMnGa," Appl. Phys. Lett., vol. 102, no. 1, p. 11912, 2013.

[21] I. Benichou and S. Givli, "Structures undergoing discrete phase transformation," J. Mech. Phys. Solids, vol. 61, no. 1, pp. 94-113, 2013.

[22] A. Cherkaev, E. Cherkaev, and L. Slepyan, "Transition waves in bistable structures. I. Delocalization of damage,” J. Mech. Phys. Solids, vol. 53, no. 2, pp. 383-405, 2005.

[23] L. Truskinovsky and A. Vainchtein, "Kinetics of martensitic phase transitions: lattice model," SIAM J. Appl. Math., vol. 66, no. 2, pp. 533-553, 2005.

[24] M. F. Ashby, T. Evans, N. A. Fleck, J. W. Hutchinson, H. N. G. Wadley, and L. J. Gibson, Metal Foams: A Design Guide: A Design Guide. Butterworth-Heinemann, 2000.

[25] Standard Test Method for Measuring Vibration-Damping Properties of Materials. ASTM E756-05. 2010.

[26] M. Carfagni, E. Lenzi, and M. Pierini, "The loss factor as a measure of mechanical damping," SPIE Proc. Ser. Soc. Photo-Optical Instrum. Eng., pp. 580-584, 1998.

[27] A. J. Wang and D. L. McDowell, "In-plane stiffness and yield strength of periodic metal honeycombs," J. Eng. Mater. Technol., vol. 126, no. 2, pp. 137-156, 2004.

[28] Y. Wang, P. Xue, and J. P. Wang, "Comparing Study of Energy-Absorbing Behavior for Honeycomb Structures," Key Eng. Mater., vol. 462-463, pp. 13-17, 2011. 
[29] L. J. Gibson and M. F. Ashby, Cellular solids: structure and properties. Cambridge university press, 1999.

[30] M. F. Ashby, Materials Selection in Mechanical Design. Butterworth-Heinemann, 2004.

[31] Z. Zheng, J. Yu, and J. Li, "Dynamic crushing of 2D cellular structures: A finite element study," Int. J. Impact Eng., vol. 32, no. 1-4, pp. 650-664, 2005.

[32] A. Honig and W. J. Stronge, "In-plane dynamic crushing of honeycomb . Part II : application to impact," vol. 44, pp. 1697-1714, 2002.

[33] A. G. Evans, M. Y. He, V. S. Deshpande, J. W. Hutchinson, A. J. Jacobsen, and W. B. Carter, "Concepts for enhanced energy absorption using hollow micro-lattices," Int. J. Impact Eng., vol. 37, no. 9, pp. 947-959, 2010.

[34] T. A. Schaedler, C. J. Ro, A. E. Sorensen, Z. Eckel, S. S. Yang, W. B. Carter, and A. J. Jacobsen, "Designing Metallic Microlattices for Energy Absorber Applications," Adv. Eng. Mater., vol. 16, no. 3, pp. 276-283, 2014. 